\title{
Equity and elderly health in India: reflections from 75th round National Sample Survey, 2017-18, amidst the COVID-19 pandemic
}

\author{
Alok Ranjan *i) and V. R. Muraleedharan(D)
}

\begin{abstract}
Background: Severe Acute Respiratory Syndrome Coronavirus-2 (SARS-CoV-2) outbreak, called coronavirus disease 2019 (COVID-19), has affected more than 200 countries across the globe with a higher fatality rate among the elderly population. Aim of the study is to highlight the vulnerability of the aged amidst the current COVID-19 pandemic, and in the light of the recent international evidence, suggests what government could do to mitigate their vulnerability.
\end{abstract}

Methods: Data from the recently released (November 2019) 75th Round National Sample Survey (NSS), which was conducted from July 2017 to June 2018, across 8077 rural villages and 6181 urban wards was used for this study. Data collected from 555,115 individuals (rural: 325,232; urban: 229,232) included 42,762 elderly individuals (60 years or above). Bivariate and multivariate analyses were used for the calculation.

Results: Of the total sample of elderly individuals, $27.7 \%$ reported suffering from an ailment in the last 15 days, whereas $8.5 \%$ had been hospitalized during the last 365 days. Among the elderly, hospitalization rate was higher in the urban areas (OR: 1.23), general social category (OR: 1.18), richest economic quintile (OR: 1.69), and among those living alone (OR: 2.40). Also, among the elderly, 64\% of those in the scheduled tribe (social group) and 51\% in the poorest economic quintile utilized public facilities for hospitalization. Cardiovascular ailments were the major cause for hospitalization (18.1\%) and outpatient visit (32\%) among the elderly. Ailments related to diabetes and hypertension constituted $55 \%$ of outpatient visit for the elderly. Only $18.9 \%$ of the elderly had health insurance though chances of facing catastrophic health expenditures were high among the elderly. $6.6 \%$ of elderly female and $1.6 \%$ male live alone, and $27.5 \%$ of age 80 years and above are immobile. $50 \%$ of male and $90 \%$ of female are financially dependent on others and more so in poorer economic quintiles.

Conclusions: The vulnerability of India's elderly increases across economic levels, and other dimensions such as the place of residence, gender, social group (caste), marital status, living arrangements, surviving children, and economic dependence. The current COVID-19 pandemic poses a greater risk of social isolation among the elderly, which may cause detrimental health impact.

Trial registration: Not applicable since the study is based on secondary data.

Keywords: Elderly heath, Equity, COVID-19, India

\footnotetext{
*Correspondence: alokranjancmc@gmail.com

Department of Humanities and Social Sciences, Indian Institute of

Technology-Madras, Chennai, India
}

(c) The Author(s). 2020 Open Access This article is licensed under a Creative Commons Attribution 4.0 International License, which permits use, sharing, adaptation, distribution and reproduction in any medium or format, as long as you give appropriate credit to the original author(s) and the source, provide a link to the Creative Commons licence, and indicate if changes were made. The images or other third party material in this article are included in the article's Creative Commons. licence, unless indicated otherwise in a credit line to the material. If material is not included in the article's Creative Commons licence and your intended use is not permitted by statutory regulation or exceeds the permitted use, you will need to obtain permission directly from the copyright holder. To view a copy of this licence, visit http://creativecommons.org/licenses/by/4.0/ The Creative Commons Public Domain Dedication waiver (http://creativecommons.org/publicdomain/zero/1.0/) applies to the data made available in this article, unless otherwise stated in a credit line to the data. 


\section{Background}

As the current Coronavirus Disease (COVID-19) pandemic spreads across the globe, the elderly population (60 years and above) become particularly vulnerable [1, 2]. Mortality data from different countries and various studies show that the elderly population is more susceptible compared to their younger counterparts $[2,3]$. However, all elderly are not equally vulnerable to COVID-19. Mortality data from South Korea show fatality rates due to COVID-19 were $1.8 \%$ in the age group of $60-69$ years, $6.3 \%$ in $70-79$ years, and $13 \%$ in the age group 80 years and above [4]. Emerging studies from the United States show that the chances of contracting COVID-19 were three times higher in black counties compared to white counties, and similarly, the death rate in black counties was six times higher than in white counties [5]. Even in India, spread and fatality of COVID-19 are significantly higher in slum areas where poor people live [6].

In this regard, it is imperative to understand the current status of the elderly health and related socioeconomic dimensions in India. This may provide valuable insights about mitigation strategies to take care of the elderly during the current COVID-19 pandemic, equitably. Recently released data (November. 2019) by the National Sample Survey (NSS) for its 75th Round, 2017-18, on social consumption related to health, provides an opportunity to understand the health status of elderly in India [7], and social support systems the elderly have across socioeconomic groups. To the best of our knowledge, this is the most recent national-level unit data on the elderly we have for India, before the outbreak of the COVID-19 pandemic. The primary aim of this study is to present the health status of the elderly in the country across various socioeconomic categories from this national level survey, and to relate it with the potential impact of COVID-19 pandemic. In the elderly population too, vulnerability varies across various dimensions such as place of residence, gender, social group (caste), occupation, income levels, living arrangements, and economic dependence. This study also aims to provide insights into interplay of these factors. This paper is organized as follows: Section II presents details of the methods used for our analysis; Section III presents results related to elderly health status, access to healthcare, financial risk protection, living arrangements, economic dependence, physical immobility, perception of self-health, and changes in the health status of elderly from 2014 to 2017-18; Section IV presents a discussion on lessons learned from these findings and offers a few suggetions for mitigating the adverse impact of the current pandemic on the elderly in India; and Section V offers a few concluding remarks.

\section{Methods}

Data for the current study is extracted from the 75th Round of NSS, which is a nation-wide sample survey conducted by the Government of India from July 2017 to June 2018 [7]. This survey covered 113,823 sample households and 555,115 individuals (Rural: 325,883; Urban: 229,232; Male: 283,200; Female: 271,877) from randomly selected 8077 villages and 6181 urban wards by two-stage random sampling method. In the first stage, rural villages and urban wards were selected, and in the second stage, households were selected. The entire sample included 42,762 elderly individuals.

The 75th Round NSS, 2017-18, collected information related to demographic details, household characteristics, morbidity and mortality, hospitalization in the last 365 days, health insurance coverage, out-ofpocket expenditure (OOPE), healthcare utilization, immunization coverage, maternal health, and elderly health [8]. Survey considerd 15 days recall period in self-reporting of acute ailments. One of the reasons for choosing 15 days recall period over monthly recall (30 days) was to reduce the recall bias in reporting the ailments. There is a higher chance of forgetfulness in monthly reporting compared to 15 days recall. Also, monthly reporting of acute ailment might erase significant fraction of relevant actions (doctors visit, expenditure on health, duration of illness etc.) taken by individuals, and more so in lower socio-economic population [8]. The current study focuses on elderly health and indicators related to this group.

For analysis purpose, age of the elderly groups were categorized as 60-69, 70-79, and 80 and above. Employment status was broadly categorized as self-employed, regular wage, casual labourer, and others. The economic quintiles for the household were assigned based on the Usual Annual per capita Consumption Expenditures (UAPCE) for rural and urban areas, respectively. UAPCE includes household expenditures other than for healthcare. It categorizes households into five economic quintiles (1-poorest, 2-poor, 3-middle, 4-rich, 5-richest). All individuals above the age of seven were categorized under broader education categories of illiterate, up to primary (8th std.), up to secondary (10th std.), and above secondary level.

Members of households were asked whether they were hospitalized in the last 365 days; and whether they suffered from any long term chronic ailment or acute ailment in the last 15 days. Data on chronic and acute ailments were used for calculating Proportion of Ailing Person (PAP) in the last 15 days.

The survey also collected information from households on whether they sought care from private or public facilities, amount of money spent for various services, including physician fees, drugs, diagnostics, and non-medical 
expenses such as travel, food etc., for both inpatient and outpatient services, and who paid for such expenses.

A total of 63 different reported ailments were broadly grouped under the following 15 ailment-categories. These are: infections, cancer, blood disease, endocrine and metabolic disease including diabetes, psychiatric and neurological, genito-urinary, eye, ear, cardio-vascular including hypertension, respiratory, gastrointestinal, skin, musculoskeletal, injuries, obstetric, and unclassified conditions.

Detailed information on how expenses were met from various sources and who paid for such expenses were obtatined for each episode of illness and service utilization. Out of pocket expenditures (OOPEs) were calculated (which is net of medical and transportation expenditures after deducting reimbursement from the insurance schemes). Catastrophic health expenditure at 10\% (CHE-10) and 25\% (CHE-25) threshold was calculated if the total annual health expenditure of the household was higher than $10 \%$ and $25 \%$, respectively, of UAPCE, based on WHO Sustainable Development Goals (SDGs) [9].

Elderly were asked additional questions about their living conditions: whether 1) Living with spouse and other members, 2) Living with spouse only, 3) Living without spouse but with children or relatives, 4) Living alone but not as an inmate of old age home, and 5) Living alone as an inmate of old age home. Financially, whether they are fully independent, partially independent, or fully dependent on others. For our analysis, partially dependent or fully dependent were considered as "dependent".

Besides, information on their physical mobility, which is an indicators of their disability [10], was recorded. In present study, "confined to bed", "confined to home", "movement on wheelchair" are considered as 'physically immobile'.

Elderly were also asked about their surviving children. We categorize them as living as "at least one living child" and "no child" for analysis purpose.

It is important to note that the elderly were asked about their self-perception of current health status, which was categorized as excellent, good, and poor. They were also asked to compare their perception of current health status with the previous year's health status, such as, 'much better', 'somewhat better', 'nearly the same', 'somewhat worse', and 'worse'. "Somewhat worse" and "worse" have been categorized as "worse" in our analysis.

Binary logistic regression was used to understand factors affecting hospitalization, PAP, CHE-10, CHE-25, living arrangements, and economic dependence in the elderly. In case of hospitalization, the dependent variable was the incidence of hospitalization, and independent variables were age group, place of residence, gender, surviving children, social group (caste), education category, household occupation, economic quintile, insurance coverage, economic independence, and living arrangements. Similarly, for the incidence of PAP, the same independent variables were used, except insurance coverage since insurance schemes do not cover outpatient care. For CHE-10 and CHE-25, other than the above mentioned independent variables, choice of provider (public or private) was included in the model. To understand factors affecting living arrangements and economic independence in the elderly population, chances of 'living alone', and chances of 'being dependent' were dependent variables, with same independent variables (except economic independence, and insurance coverage in living arrangement model; and household occupation, and insurance coverage in financial dependent model) mentioned above for the logit model of hospitalization. These variables have been included based on suggestions from existing literature [11-16]. It is important to note here that the literature also suggests that "marital status" be considered as an explanatory variable. We have dropped this factor as it showed a high multicollinearity (Variation Inflation Factor: 23). No multicollinearity was found among independent variables used in our analysis (refer Additional File 1).

Findings of the 75th Round NSS, 2017-18, were also compared with 71st Round NSS, 2014, for similar indicators, to understand the change in state of elderly health from 2014 to 2017-18. Bivariate and multivariate analyses were performed using STATA version 14.1.

\section{Results}

Findings of the study are presented under eight themes: 1) Demographic characteristics, 2) Disease burden and access to healthcare, 3) Financial hardship, 4) Living arrangements, 5) Economic dependence, 6) Physical immobility, 7) Perception towards own health, and 8) Change in health status of the elderly from 2014 (71st Round NSS) to 2017-18 (75th Round NSS). All observations relate to the elderly population, unless stated otherwise.

\section{Demographic characteristics}

The average age of the elderly population in India was 67.5 years (Table 1). Out of total elderly population, $66.1 \%$ are in the age group of $60-69$ years, $25.9 \%$ in 70 79 , and $8 \%$ are aged 80 years and above. $67.1 \%$ of India's elderly live in rural areas. Proportion of female (50.9\%) is higher than male (49.1\%). In terms of the social groups, $6.2 \%$ elderly belongs to scheduled tribe (ST) category, $17.4 \%$ scheduled caste (SC) category, $42.3 \%$ to other backward class $(\mathrm{OBC})$, and $34.3 \%$ belongs to general category. In India, $54.1 \%$ of the elderly people are illiterate, 
Table 1 Demographic and Socio-economic characteristics of sample elderly population in India

\begin{tabular}{lll}
\hline & India & Sample size (N) \\
\hline $\begin{array}{l}\text { Mean Age (years) } \\
\text { Age group (years) }\end{array}$ & 67.5 & 42,762 \\
$60-69$ & 66.1 & \\
$70-79$ & 27,769 \\
80 and above & 8.0 & 11,235 \\
\end{tabular}

Place of Residence

$\begin{array}{lll}\text { Rural } & 67.1 & 23,599 \\ \text { Urban } & 32.9 & 19,163 \\ \text { Gender } & & \\ \text { Male } & 49.1 & 21,902 \\ \text { Female } & 50.9 & 20,858\end{array}$

Marital Status

$\begin{array}{lll}\text { Never Married/divorced/separated } & 0.9 & 395 \\ \text { Currently married } & 64.7 & 29,324 \\ \text { Widowed } & 34.4 & 13,043\end{array}$

Surviving Children

$\begin{array}{lll}\text { At least one surviving child } \quad 95.7 & 41,409\end{array}$

No child

$4.3 \quad 1353$

Social Groups

ST

SC

$\mathrm{OBC}$

General

Education

Illiterate

Up to primary

Up to secondary

Above Secondary

Household occupation

Self employed

Regular Wages

Casual Labourer

Others

\begin{tabular}{ll}
6.2 & 3913 \\
17.4 & 6133 \\
42.3 & 16,519 \\
34.3 & 16,197 \\
& \\
54.1 & 20,194 \\
21.1 & 9375 \\
14.3 & 7752 \\
10.5 & 5441 \\
& \\
48.1 & 20,986 \\
15.5 & 8536 \\
20.0 & 6709 \\
16.5 & 6531 \\
& \\
19.1 & 3773 \\
19.0 & 3945 \\
21.0 & 4651 \\
19.5 & 4949 \\
21.3 & 6281 \\
21.7 & 4776 \\
18.0 & 3614 \\
20.9 & 3696 \\
& \\
\hline 10.5 \\
\end{tabular}

Table 1 Demographic and Socio-economic characteristics of sample elderly population in India (Continued)

\begin{tabular}{lll}
\hline & India & Sample size (N) \\
\hline Rich & 22.0 & 3537 \\
Richest & 17.3 & 3540 \\
\hline Source: Authors' computation from unit records of NSSO & 75th Round 2017-18
\end{tabular}

and $20 \%$ belongs to those households where casual labor is the main household occupation. Also, $4.3 \%$ of the elderly in India do not have a surviving child (Table 1).

\section{Disease-burden and access to healthcare Outpatient care}

Out of every 100 elderly, 27.7 pesons reported ailments during the previous 15 days; of this, 22.4 reported chronic ailments, and 5.7 reported acute ailments (Table 2). PAP was significantly high among 80 years and above (36.7\%), those in urban areas (34.0\%), widowed (30.8\%), general category $(33.2 \%)$, having regular wages $(31.5 \%)$ and in the richest economic quintile (rural-36.8\%, urban-43.8\%, Table 2, Table 3). The logistic model shows that chances of reporting ailment in last 15 days was 1.50 times higher among 80 years and above compared to those in age group 60-69 years; 1.44 times higher in urban areas compared to rural areas, 2.08 times higher in general category compared to lower socio-economic groups (ST category), 1.33 times higher among those with primary level education compared to illiterate, 1.23 times higher among casual labourers compared to regular wage earners, and 2.47 times higher among those in the richest economic quintile compared to their poorest counterparts (Table 3).

Cardiovascular conditions including hypertension (32.0\%), endocrine conditions including diabetes (22.5\%), musculoskeletal conditions (13.9\%), infectious diseases $(10.0 \%)$, and respiratory ailments $(7.3 \%)$ were the topfive conditions for seeking outpatient care among the elderly in the last 15 days (Table 4). 33.6\% of the elderly went to a public provider in the last 15 days, particularly for cancer (55.8\%) and eye-related problems (47.5\%, Table 4). Public healthcare utilization was higher in rural areas (39.7\%), ST category (43.5\%), casual labourer (50.3\%), illiterate $(37.7 \%)$, never married or divorced $(47.6 \%)$ and poorest economic quintile compared to their respective counterparts (rural- $45.3 \%$, urban- $41.6 \%$, Table 2).

\section{Inpatient care}

Overall hospitalization rate among the elderly was $8.5 \%$, and was highest among 80 years and above (14.3\%) - it was significantly high for male $(9.5 \%)$, those in urban areas $(10.1 \%)$, never married/divorced (8.8\%), those with no surviving children (21.3\%), in general social category $(10.0 \%)$, regular wages earners $(9.2 \%)$, and those in the richest economic quintile (rural-11.2\%, urban-11\%- 
Table 2 Disease burden and access to healthcare in elderly population of India

\begin{tabular}{|c|c|c|c|c|c|c|}
\hline & \multicolumn{2}{|c|}{ Hospitalization (in \%) } & \multicolumn{4}{|l|}{ Out-patient care (in \%) } \\
\hline & $\begin{array}{l}\text { Hospitalization } \\
\text { rate }\end{array}$ & $\begin{array}{l}\text { Share of } \\
\text { hospitalization } \\
\text { under public sector }\end{array}$ & $\begin{array}{l}\text { Proportion of population } \\
\text { reporting chronic } \\
\text { condition }\end{array}$ & $\begin{array}{l}\text { Proportion of population } \\
\text { reporting ailment in last } 15 \\
\text { days }\end{array}$ & $\begin{array}{l}\text { PAP in } \\
\text { last } 15 \\
\text { days }\end{array}$ & $\begin{array}{l}\text { Share of PAP } \\
\text { under public } \\
\text { sector }\end{array}$ \\
\hline Total & 8.5 & 39.8 & 22.4 & 5.7 & 27.7 & 33.6 \\
\hline \multicolumn{7}{|c|}{ Age group (years) } \\
\hline $60-69$ & 7.1 & 40.4 & 20.3 & 5.2 & 25.1 & 34.0 \\
\hline $70-79$ & 10.1 & 40.8 & 25.3 & 6.8 & 31.7 & 33.2 \\
\hline $\begin{array}{l}80 \text { and } \\
\text { above }\end{array}$ & 14.3 & 35.5 & 31.2 & 6.8 & 36.7 & 32.2 \\
\hline \multicolumn{7}{|c|}{ Place of Residence } \\
\hline Rural & 7.7 & 44.5 & 19.0 & 6.0 & 24.6 & 39.7 \\
\hline Urban & 10.1 & 32.7 & 29.5 & 5.2 & 34.0 & 25.5 \\
\hline \multicolumn{7}{|l|}{ Gender } \\
\hline Male & 9.5 & 40.3 & 22.2 & 5.8 & 27.5 & 33.5 \\
\hline Female & 7.5 & 39.3 & 22.6 & 5.7 & 27.9 & 33.7 \\
\hline \multicolumn{7}{|l|}{ Marital Status } \\
\hline $\begin{array}{l}\text { Never } \\
\text { Married/ } \\
\text { divorced/ }\end{array}$ & 8.8 & 41.8 & 23.1 & 2.2 & 25.0 & 47.6 \\
\hline $\begin{array}{l}\text { Currently } \\
\text { married }\end{array}$ & 7.8 & 38.1 & 21.2 & 5.3 & 26.1 & 32.1 \\
\hline Widowed & 7.7 & 40.4 & 24.8 & 6.5 & 30.8 & 35.7 \\
\hline \multicolumn{7}{|c|}{ Surviving Children } \\
\hline $\begin{array}{l}\text { At least } \\
\text { one } \\
\text { surviving } \\
\text { child }\end{array}$ & 7.8 & 38.9 & 22.5 & 5.8 & 27.9 & 33.5 \\
\hline No child & 21.3 & 46.0 & 19.9 & 4.5 & 24.2 & 37.0 \\
\hline \multicolumn{7}{|l|}{ Social Groups } \\
\hline ST & 5.5 & 64.0 & 10.6 & 7.3 & 17.8 & 43.5 \\
\hline SC & 7.5 & 53.2 & 18.4 & 6.5 & 24.7 & 38.6 \\
\hline $\mathrm{OBC}$ & 8.2 & 38.4 & 20.9 & 5.6 & 26.0 & 42.2 \\
\hline General & 10.0 & 33.8 & 28.5 & 5.3 & 33.2 & 22.7 \\
\hline \multicolumn{7}{|l|}{ Education } \\
\hline Illiterate & 6.1 & 46.1 & 17.8 & 5.9 & 23.4 & 37.7 \\
\hline $\begin{array}{l}\text { Up to } \\
\text { primary }\end{array}$ & 10.3 & 42.8 & 26.5 & 6.8 & 32.6 & 41.2 \\
\hline $\begin{array}{l}\text { Up to } \\
\text { secondary }\end{array}$ & 9.7 & 29.1 & 30.4 & 4.9 & 34.5 & 26.7 \\
\hline $\begin{array}{l}\text { Above } \\
\text { Secondary }\end{array}$ & 8.0 & 17.4 & 27.5 & 3.7 & 30.8 & 15.3 \\
\hline \multicolumn{7}{|c|}{ Household occupation } \\
\hline $\begin{array}{l}\text { Self } \\
\text { employed }\end{array}$ & 7.1 & 36.5 & 20.1 & 6.0 & 25.8 & 31.0 \\
\hline $\begin{array}{l}\text { Regular } \\
\text { Wages }\end{array}$ & 9.2 & 37.4 & 25.7 & 6.2 & 31.5 & 27.1 \\
\hline $\begin{array}{l}\text { Casual } \\
\text { Labourer }\end{array}$ & 6.5 & 53.8 & 18.6 & 5.8 & 24.0 & 50.3 \\
\hline
\end{tabular}


Table 2 Disease burden and access to healthcare in elderly population of India (Continued)

\begin{tabular}{|c|c|c|c|c|c|c|}
\hline & \multicolumn{2}{|c|}{ Hospitalization (in \%) } & \multicolumn{4}{|l|}{ Out-patient care (in \%) } \\
\hline & $\begin{array}{l}\text { Hospitalization } \\
\text { rate }\end{array}$ & $\begin{array}{l}\text { Share of } \\
\text { hospitalization } \\
\text { under public sector }\end{array}$ & $\begin{array}{l}\text { Proportion of population } \\
\text { reporting chronic } \\
\text { condition }\end{array}$ & $\begin{array}{l}\text { Proportion of population } \\
\text { reporting ailment in last } 15 \\
\text { days }\end{array}$ & $\begin{array}{l}\text { PAP in } \\
\text { last } 15 \\
\text { days }\end{array}$ & $\begin{array}{l}\text { Share of PAP } \\
\text { under public } \\
\text { sector }\end{array}$ \\
\hline \multicolumn{7}{|c|}{ Economic quintile-Rural } \\
\hline Poorest & 5.0 & 51.0 & 9.6 & 7.3 & 16.7 & 45.3 \\
\hline Poor & 5.0 & 52.2 & 15.8 & 5.9 & 21.5 & 34.9 \\
\hline Middle & 6.7 & 47.1 & 15.6 & 6.2 & 21.6 & 39.1 \\
\hline Rich & 8.3 & 44.3 & 20.0 & 5.6 & 25.3 & 39.4 \\
\hline Richest & 11.2 & 37.7 & 32.7 & 5.1 & 36.8 & 40.5 \\
\hline \multicolumn{7}{|c|}{ Economic quintile-Urban } \\
\hline Poorest & 9.7 & 47.7 & 22.5 & 4.7 & 26.7 & 41.6 \\
\hline Poor & 9.7 & 41.6 & 28.4 & 3.4 & 31.4 & 31.5 \\
\hline Middle & 9.7 & 35.1 & 27.3 & 8.3 & 35.1 & 27.1 \\
\hline Rich & 10.1 & 26.4 & 30.8 & 4.8 & 34.7 & 22.5 \\
\hline Richest & 11.0 & 14.4 & 40.2 & 4.3 & 43.8 & 12.5 \\
\hline
\end{tabular}

Source: Authors' computation from unit records of NSSO 75th Round 2017-18

Table 2 and Table 3). Cardiovascular diseases (18.1\%), infectious diseases $(16.6 \%)$, eye ailments $(8.4 \%)$, psychiatric or neurological conditions (8.2\%), and injuries (7.9\%) were the top five reasons for hospitalization in the last 365 days (Table 4).

Public facilities accounted for $39.8 \%$ of all inpatient services availed by the elderly - it was higher in rural areas (44.5\%), those with no surviving children (46.0\%), ST category $(64.0 \%)$, illiterate $(46.1 \%)$, and poorest income quintile compared to respective counterparts (rural-51.0\%, urban-47.7\%, Table 2). Share of the public sector was higher for cancer treatment $(52.8 \%)$, skin related ailments (50.3\%), infectious diseases (48.3\%), and blood diseases $(46.8 \%)$ whereas the share of the private sector was higher for most other conditions. For instance, private facilities accounted for $72.2 \%$ of genitourinary, $63.2 \%$ of psychiatric and neurological conditions, and $63.1 \%$ of injury related inpatient care (Table 4 ).

\section{Financial risk protection}

Publicly funded health insurance (PFHI) coverage and (tax funded) subsidized public provisioning are the two major strategies used by the government for providing financial risk protection in India [11].

\section{Health insurance}

Overall, $18.9 \%$ of the elderly were coved under health insurance schemes; whereas PFHIs covered $14.3 \%$ population. PFHIs only cover inpatient care in India, whereas Central Government Health Schemes (CGHS-2.1\%) and ESIS $(0.7 \%)$ cover outpatient care as well. Private insurance also provided coverage $(1.8 \%)$, but for inpatient care alone [7]. PFHIs coverage among the elderly was high in rural areas (16.6\%), ST category (20.7\%), illiterate (16.6\%), casual labourer (18.2\%), and poorest rural quintile $(12.8 \%)$ in India. Insurance coverage in urban areas was more equitable compared to rural areas, since PFHI coverage was higher in poorer quintile compared to richer quintile in urban elderly. In the rural areas, PFHI coverage was higher in top two quintiles compared to the bottom two quintiles (Table 5).

\section{Outpatient care}

OOPE for outpatient care was Rs. 390 per visit under public sector, and Rs.852 per visit under private sector (Table 5). OOPE was significantly higher for those 80 years and above (public: 430, private: 1039). OOPE was almost the same under rural and urban India. OOPE was higher for male compared to the female gender in the public sector, whereas under the private sector it was nearly the same. OOPE was higher in ST category population compared to general category population in both public and private sectors. The public sector was more equitable compared to the private sector. For instance, under public sector, OOPE was Rs. 371 for the poorest income quintile as Rs.564 for the richest quintile. On the other hand, under the private sector, OOPE was Rs. 995 for the poorest quintile and Rs. 916 for the richest quintile.

\section{Inpatient care}

Average OOPE was Rs. 6209 under public sector and Rs. 38,709 under private sector. OOPE was significantly higher for male, urban areas, never married or divorced, elderly without children, ST category, above secondary 
Table 3 Factors affecting hospitalization, PAP, CHE-10/25, living arrangements, and economic dependence in India's elderly

\begin{tabular}{|c|c|c|c|c|c|c|}
\hline Total & $\begin{array}{l}\text { Reporting of } \\
\text { hospitalization } \\
\text { OR }(95 \% \mathrm{CI})\end{array}$ & $\begin{array}{l}\text { Reporting } \\
\text { of PAP } \\
\text { OR }(95 \% \mathrm{CI})\end{array}$ & $\begin{array}{l}\text { CHE-10 } \\
\text { OR }(95 \% \\
\text { Cl) }\end{array}$ & $\begin{array}{l}\text { CHE-25 } \\
\text { OR }(95 \% \\
\text { Cl) }\end{array}$ & $\begin{array}{l}\text { Living } \\
\text { alone } \\
\text { OR }(95 \% \mathrm{Cl})\end{array}$ & $\begin{array}{l}\text { Economically } \\
\text { dependent } \\
\text { OR }(95 \% \mathrm{Cl})\end{array}$ \\
\hline \multicolumn{7}{|c|}{ Age group (years, ref:60-69) } \\
\hline $70-79$ & $1.33(1.27-1.40)^{*}$ & $\begin{array}{l}1.25(1.19- \\
1.32)^{*}\end{array}$ & $\begin{array}{l}1.02(0.93- \\
1.13)\end{array}$ & $\begin{array}{l}1.07(0.96- \\
1.19)\end{array}$ & $\begin{array}{l}1.02(0.86- \\
1.21)\end{array}$ & $1.93(1.82-2.04)^{*}$ \\
\hline 80 and above & $1.37(1.27-1.48)^{*}$ & $\begin{array}{l}1.50(1.39- \\
1.61)^{*}\end{array}$ & $\begin{array}{l}1.00(0.87- \\
1.16)\end{array}$ & $\begin{array}{l}0.99(0.84- \\
1.16)\end{array}$ & $\begin{array}{l}0.86(0.65- \\
1.12)\end{array}$ & $3.59(3.23-3.99)^{*}$ \\
\hline \multicolumn{7}{|c|}{ Place of Residence (ref: rural) } \\
\hline Urban & $1.23(1.16-1.29)^{*}$ & $\begin{array}{l}1.44(1.37- \\
1.51)^{*}\end{array}$ & $\begin{array}{l}0.50(0.45- \\
0.56)^{*}\end{array}$ & $\begin{array}{l}0.53(0.47- \\
0.59)^{*}\end{array}$ & $\begin{array}{l}0.86(0.72- \\
1.02)\end{array}$ & $0.98(0.92-1.03)$ \\
\hline \multicolumn{7}{|l|}{ Gender (ref: male) } \\
\hline Female & $0.69(0.65-0.72)^{*}$ & $\begin{array}{l}0.98(0.93- \\
1.03)\end{array}$ & $\begin{array}{l}0.79(0.71- \\
0.87)^{*}\end{array}$ & $\begin{array}{l}0.78(0.70- \\
0.88)^{*}\end{array}$ & $\begin{array}{l}2.82(2.38- \\
3.35)^{*}\end{array}$ & $10.13(9.53-10.8)^{*}$ \\
\hline \multicolumn{7}{|c|}{ Surviving Children (ref: no child) } \\
\hline At least one child & $0.97(0.85-1.09)$ & $\begin{array}{l}1.32(1.16- \\
1.50)^{*}\end{array}$ & $\begin{array}{l}0.75(0.60- \\
0.95)\end{array}$ & $\begin{array}{l}0.81(0.64- \\
1.03)\end{array}$ & $\begin{array}{l}0.24(0.19- \\
0.30)^{*}\end{array}$ & $2.06(1.80-2.36)^{*}$ \\
\hline \multicolumn{7}{|c|}{ Social Groups (ref: ST) } \\
\hline SC & $1.09(0.99-1.20)$ & $\begin{array}{l}1.81(1.63- \\
2.02)^{*}\end{array}$ & $\begin{array}{l}1.35(1.10- \\
1.66)^{* *}\end{array}$ & $\begin{array}{l}1.55(1.19- \\
2.00)^{* *}\end{array}$ & $\begin{array}{l}1.28(0.92- \\
1.79)\end{array}$ & $1.06(0.95-1.17)$ \\
\hline $\mathrm{OBC}$ & $1.16(1.07-1.27)^{* *}$ & $\begin{array}{l}1.89(1.71- \\
2.08)^{*}\end{array}$ & $\begin{array}{l}1.46(1.21- \\
1.75)^{*}\end{array}$ & $\begin{array}{l}1.68(1.33- \\
2.11)^{*}\end{array}$ & $\begin{array}{l}1.22(0.90- \\
1.64)\end{array}$ & $1.20(1.09-1.31)^{*}$ \\
\hline General & $1.18(1.08-1.29)^{*}$ & $\begin{array}{l}2.08(1.89- \\
2.29)^{*}\end{array}$ & $\begin{array}{l}1.41(1.17- \\
1.69)^{*}\end{array}$ & $\begin{array}{l}1.59(1.26- \\
2.01)^{*}\end{array}$ & $\begin{array}{l}1.22(0.90- \\
1.65)\end{array}$ & $1.19(1.08-1.30)^{*}$ \\
\hline \multicolumn{7}{|c|}{ Education (ref: illiterate) } \\
\hline Up to primary & $1.09(1.03-1.16)^{* *}$ & $\begin{array}{l}1.33(1.26- \\
1.41)^{*}\end{array}$ & $\begin{array}{l}1.18(1.05- \\
1.33)^{* *}\end{array}$ & $\begin{array}{l}1.26(1.11- \\
1.44)^{*}\end{array}$ & $\begin{array}{l}0.73(0.60- \\
0.90)^{* *}\end{array}$ & $0.85(0.79-0.91)^{*}$ \\
\hline Up to secondary & $0.98(0.92-1.05)$ & $\begin{array}{l}1.17(1.10- \\
1.25)^{*}\end{array}$ & $\begin{array}{l}1.40(1.22- \\
1.60)^{*}\end{array}$ & $\begin{array}{l}1.50(1.30- \\
1.73)^{*}\end{array}$ & $\begin{array}{l}0.36(0.28- \\
0.46)^{*}\end{array}$ & $0.54(0.50-0.58)^{*}$ \\
\hline Above Secondary & $0.91(0.84-0.99)^{* *}$ & $\begin{array}{l}1.02(0.94- \\
1.11)\end{array}$ & $\begin{array}{l}1.48(1.26- \\
1.74)^{*}\end{array}$ & $\begin{array}{l}1.60(1.34- \\
1.90)^{*}\end{array}$ & $\begin{array}{l}0.34(0.26- \\
0.45)^{*}\end{array}$ & $0.26(0.24-0.28)^{*}$ \\
\hline \multicolumn{7}{|c|}{ Household occupation (ref: self-employed) } \\
\hline Regular Wages & $1.02(0.95-1.08)$ & $\begin{array}{l}1.12(1.05- \\
1.19)^{*}\end{array}$ & $\begin{array}{l}1.01(0.89- \\
1.14)\end{array}$ & $\begin{array}{l}0.93(0.81- \\
1.08)\end{array}$ & $\begin{array}{l}0.49(0.28- \\
0.87)^{* *}\end{array}$ & NA \\
\hline Casual Labourer & $1.03(0.96-1.10)$ & $\begin{array}{l}1.23(1.15- \\
1.32)^{*}\end{array}$ & $\begin{array}{l}0.99(0.86- \\
1.13)\end{array}$ & $\begin{array}{l}1.13(0.97- \\
1.32)\end{array}$ & $\begin{array}{l}3.01(2.21- \\
4.09)^{*}\end{array}$ & NA \\
\hline \multicolumn{7}{|c|}{ Economic quintile (ref: poorest) } \\
\hline Poor & $1.11(1.03-1.20)^{* *}$ & $\begin{array}{l}1.29(1.19- \\
1.39)^{*}\end{array}$ & $\begin{array}{l}0.63(0.53- \\
0.73)^{*}\end{array}$ & $\begin{array}{l}0.63(0.53- \\
0.74)^{*}\end{array}$ & $\begin{array}{l}0.60(0.45- \\
0.81)^{* *}\end{array}$ & $0.96(0.88-1.04)$ \\
\hline Middle & $1.31(1.22-1.41)^{*}$ & $\begin{array}{l}1.56(1.44- \\
1.68)^{*}\end{array}$ & $\begin{array}{l}0.56(0.48- \\
0.64)^{*}\end{array}$ & $\begin{array}{l}0.59(0.50- \\
0.69)^{*}\end{array}$ & $\begin{array}{l}0.79(0.61- \\
1.03)\end{array}$ & $0.90(0.83-0.97)^{* *}$ \\
\hline Rich & $1.44(1.38-1.55)^{*}$ & $\begin{array}{l}1.68(1.56- \\
1.81)^{*}\end{array}$ & $\begin{array}{l}0.47(0.40- \\
0.54)^{*}\end{array}$ & $\begin{array}{l}0.51(0.43- \\
0.60)^{*}\end{array}$ & $\begin{array}{l}1.11(0.87- \\
1.42)\end{array}$ & $0.80(0.74-0.87)^{*}$ \\
\hline Richest & $1.69(1.56-1.82)^{*}$ & $\begin{array}{l}2.47(2.29- \\
2.66)^{*}\end{array}$ & $\begin{array}{l}0.36(0.31- \\
0.42)^{*}\end{array}$ & $\begin{array}{l}0.40(0.34- \\
0.47)^{*}\end{array}$ & $\begin{array}{l}1.38(1.09- \\
1.75)\end{array}$ & $0.71(0.65-0.77)^{*}$ \\
\hline \multicolumn{7}{|c|}{ Insurance coverage (ref: No) } \\
\hline Yes & $1.27(1.20-1.34)^{*}$ & NA & $\begin{array}{l}0.52(0.47- \\
0.58)^{*}\end{array}$ & $\begin{array}{l}0.57(0.50- \\
0.64)^{*}\end{array}$ & NA & NA \\
\hline \multicolumn{7}{|l|}{ Provider (ref: public) } \\
\hline Private & NA & NA & $\begin{array}{l}8.18(7.41- \\
9.02)^{*}\end{array}$ & $\begin{array}{l}7.52(6.6- \\
8.51)^{*}\end{array}$ & NA & NA \\
\hline \multicolumn{7}{|c|}{ Economic independence (ref: independent) } \\
\hline Dependent & $1.39(1.31-1.47)^{*}$ & $1.26(1.19-$ & $1.06(0.95-$ & $0.94(0.83-$ & NA & NA \\
\hline
\end{tabular}


Table 3 Factors affecting hospitalization, PAP, CHE-10/25, living arrangements, and economic dependence in India's elderly (Continued)

\begin{tabular}{|c|c|c|c|c|c|c|}
\hline Total & $\begin{array}{l}\text { Reporting of } \\
\text { hospitalization } \\
\text { OR }(95 \% \mathrm{Cl})\end{array}$ & $\begin{array}{l}\text { Reporting } \\
\text { of PAP } \\
\text { OR }(95 \% \mathrm{Cl})\end{array}$ & $\begin{array}{l}\text { CHE-10 } \\
\text { OR }(95 \% \\
\text { Cl) }\end{array}$ & $\begin{array}{l}\text { CHE- } 25 \\
\text { OR }(95 \% \\
\text { Cl) }\end{array}$ & $\begin{array}{l}\text { Living } \\
\text { alone } \\
\text { OR }(95 \% \mathrm{Cl})\end{array}$ & $\begin{array}{l}\text { Economically } \\
\text { dependent } \\
\text { OR }(95 \% \mathrm{Cl})\end{array}$ \\
\hline & & $1.33)^{*}$ & 1.18) & 1.06) & & \\
\hline \multicolumn{7}{|l|}{ Living arrangement (ref: with spouse/family) } \\
\hline Living alone & $2.40(2.07-2.78)^{*}$ & $\begin{array}{l}1.66(1.43- \\
1.93)^{*}\end{array}$ & $\begin{array}{l}2.07(1.62- \\
2.64)^{*}\end{array}$ & $\begin{array}{l}2.04(1.59- \\
2.62)^{*}\end{array}$ & NA & $0.16(0.14-0.19)^{*}$ \\
\hline Constant & $0.16(0.14-0.19)^{*}$ & $\begin{array}{l}0.05(0.04- \\
0.06)^{*}\end{array}$ & $\begin{array}{l}0.56(0.41- \\
0.76)^{*}\end{array}$ & $\begin{array}{l}0.14(0.10- \\
0.19)^{*}\end{array}$ & $\begin{array}{l}0.009(0.006- \\
0.014)^{*}\end{array}$ & $0.64(0.54-0.75)^{*}$ \\
\hline \multicolumn{7}{|l|}{ Model Details } \\
\hline Log likelihood & $-23,767.779$ & $-24,171.993$ & $-\overline{6115.6486}$ & -5128.930 & -2938.437 & $-19,561.532$ \\
\hline Number of observations & 42,755 & 42,755 & 10,801 & 10,801 & 42,760 & 42,755 \\
\hline LR Chi2 & 1369.42 & 1974.30 & 2655.74 & 1793.75 & 2396.66 & $12,375.97$ \\
\hline Prob $>$ Chi2 & 0.000 & 0.000 & 0.000 & 0.000 & 0.000 & 0.0000 \\
\hline Pseudo R2 & 0.028 & 0.039 & 0.178 & 0.149 & 0.2897 & 0.2403 \\
\hline Mean Variance inflation factor & 1.56 & 1.58 & 1.65 & 1.65 & 1.63 & 1.620 \\
\hline Mean Pregibon dbeta & 0.258 & 0.275 & 0.45 & 0.245 & 0.019 & 0.709 \\
\hline $\begin{array}{l}\text { Specification error (linktest): predicted value } \\
\text { (_hat) }[p>|z|]\end{array}$ & 0.000 & 0.000 & 0.000 & 0000 & 0.000 & 0.000 \\
\hline $\begin{array}{l}\text { Specification error (linktest): predicted value } \\
\text { squared (_hatsq) [p }>|z|]\end{array}$ & 0.315 & 0.141 & 0.369 & 0.314 & 0.387 & 0.081 \\
\hline \multicolumn{7}{|c|}{$\begin{array}{l}\text { Note: } \\
\text { (*) } p \text {-value }<0.001 \\
\text { (**) } p \text {-value }<0.05 \\
\text { 'NA' indicates particular variable was not included the respective model } \\
\text { All estimates, except model details, are odds ratio (OR) and values in the }\end{array}$} \\
\hline
\end{tabular}

literate, and richest quintiles compared to their respective counterparts. For instance, OOPE for the poorest rural quintile was Rs. 5084 in public and Rs. 19,410 in private provider; whereas it was Rs. 7949 and Rs. 39,683 in the richest rural quintile, respectively (refer Table 5). CHE-10 and CHE-25 were calculated to estimate the impact of OOPE on the households. $23.2 \%$ of inpatients in public sector faced CHE-10, whereas $64.9 \%$ faced CHE-10 under private sector. Similarly, CHE-25 was $9.1 \%$ under public sector, and $37.0 \%$ under private sector. CHE-10 and CHE-25 were higher among those in 60-69 years age group, rural areas, male gender, never-married individuals, ST category, casual labourer, and poorest income quintile compared to their counterparts (Table 5). Chances of facing CHE-10 and CHE-25 were statistically higher for rural areas, male gender, those with no surviving children, in the poorest quintile, non-insured population, who used private provider and among the elderly living alone (Table 3). Similarly, chances of facing CHE-10 and CHE-25 was 8.18 and 7.52 times higher, respectively, under the private sector compared to the public sector.

\section{Living arrangements}

$4.2 \%$ of the elderly population lived alone, whereas $14.1 \%$ lived with spouse only (Table 6). The elderly population living alone was higher in rural areas (4.4\%), female gender $(6.6 \%)$, never married or divorced individuals (22.2\%), elderly with no surviving child (16.1\%), illiterate population (5.0\%), and the richest income quintile (rural-7.0\%, urban-6.5\%). Also, those living with their spouse were higher in the top two income quintiles compared to the bottom two quintiles. Chances of living alone was higher in female (OR: 2.82), and richest income quintile (OR: 1.87- refer Table 3).

\section{Economic dependence}

In India, $47 \%$ of elderly were financially depenedent on others; $30.1 \%$ were independent, and $22.9 \%$ were partially dependent (Table 6). In other words, $70 \%$ of India's elderly were, partially or entirely, financially dependent on others. Complete financial dependence was higher among those 80 years and above $(70.8 \%)$, female (67.1\%), widowed (62.8\%), illiterate (55.5\%), and poorer quintiles. Chances of being economically dependent were higher among 80 years and above (OR: 3.59), female (OR: 
Table 4 Disease burden and health seeking behavior in elderly during hospitalization and out-patient care in India

\begin{tabular}{|c|c|c|c|c|}
\hline & \multicolumn{2}{|l|}{ Hospitalization } & \multicolumn{2}{|l|}{ Out-patient care } \\
\hline & $\begin{array}{l}\text { Diseases burden } \\
\text { during } \\
\text { hospitalization }\end{array}$ & $\begin{array}{l}\text { Share of hospitalization episodes } \\
\text { treated under public sector }\end{array}$ & $\begin{array}{l}\text { Diseases burden in } \\
\text { out-patient care }\end{array}$ & $\begin{array}{l}\text { Share of out-patient care } \\
\text { treated under public sector }\end{array}$ \\
\hline Infection & 16.6 & 48.3 & 10.0 & 33.5 \\
\hline Cancers & 4.6 & 52.8 & 0.5 & 55.8 \\
\hline Blood diseases & 0.9 & 46.8 & 0.9 & 15.3 \\
\hline $\begin{array}{l}\text { Endocrine, metabolic, } \\
\text { nutritional (includes } \\
\text { diabetes) }\end{array}$ & 5.3 & 40.3 & 22.5 & 35.5 \\
\hline Psychiatric and Neurological & 8.2 & 36.8 & 4.4 & 28.2 \\
\hline Genito-urinary & 5.3 & 27.8 & 1.0 & 33.9 \\
\hline Eye & 8.4 & 37.8 & 1.5 & 47.5 \\
\hline Ear & 0.2 & 36.4 & 0.4 & 35.6 \\
\hline $\begin{array}{l}\text { Cardio-vascular (includes } \\
\text { hypertension) }\end{array}$ & 18.1 & 37.7 & 32.0 & 33.4 \\
\hline Respiratory & 7.8 & 45.3 & 7.3 & 37.5 \\
\hline Gastro-Intestinal & 7.5 & 36.7 & 2.6 & 30.3 \\
\hline Skin & 0.7 & 50.3 & 0.9 & 22.7 \\
\hline Musculo-skeletal & 6.2 & 34.8 & 13.9 & 33.0 \\
\hline Injuries & 7.9 & 34.9 & 0.7 & 31.1 \\
\hline Others & 2.4 & 31.4 & 1.6 & 15.3 \\
\hline Total & 100.0 & 39.8 & 100.0 & 33.6 \\
\hline
\end{tabular}

Source: Authors' computation from unit records of NSSO 75th Round 2017-18

10.13), general category population (OR: 1.19), elderly not living alone, and poorer quintiles (refer Table 3).

\section{Physical immobility}

Physical mobility is one of the proxy indicators for locomotor disability. In India, $7.6 \%$ of the elderly were either completely (bedridden) or partially immobile (on a wheelchair or restricted within their home). It was considerably high among those 80 years and above (27.5\%), female gender $(8.9 \%)$, widowed $(11.9 \%)$, illiterate population $(8.7 \%)$, and poorer income quintiles. However, immobility increases steeply for the richest quintile (rural8.3\%, urban- 9.5\%) compared to other quintiles (refer Table 6).

\section{Perception of self-health}

In India, one in five elderly (19.6\%) felt their current health status was poorer, and a similar proportion (21.0\%) felt that their health condition had deteriorated compared to the previous year (Table 6). Perception of health being poor was high among those above 80 of years, in rural areas (21.4\%), and among the widowed elderly (26\%). About $20 \%$ of those in poorest urban quintile perceived their health had deteriorated compared to the previous year (refer Table 6).
Change in the health status of the elderly in NSS 75th round, 2017-18, compared to NSS 71st round, 2014 Hospitalization rate among elderly fell from $10.9 \%$ in 2014 to $8.5 \%$ in $2017-18$ (Table 7 ). Also, PAP fell from 30.3 (out of 100 reported elder persons) in 2014 to 27.7 in 2017-18. Share of the public sector in outpatient care increased from $28.3 \%$ in 2014 , to $33.6 \%$ in $2017-18$, whereas its share in inpatient care increased from $35.9 \%$ to $39.0 \%$. OOPEs under public sector fell from Rs. 547 (in 2014) to Rs. 390 (in 2017-18) per visit for outpatient care and from Rs. 7177 (in 2014) to Rs 6209 (in 201718) per visit for hospitalization. On the other hand, OOPEs under private sector increased from Rs. 802 (in 2014) to Rs 852 (in 2017-18) per outpatient visit, and Rs. 31,875 (in 2014) to Rs. 38,709 (in 2017-18) per hospitalization visit(refer Table 7).

\section{Discussion}

Here we highlight some key findings and discuss the extent to which the elderly population is vulnerable in the light of the emerging international literature and evidence.

Disease burden in the elderly population (PAP: $27.7 \%$, hospitalization rate: $8.5 \%$ - Table 2 ) is disproportionately higher compared to the population below the age of 60 
Table 5 Financial Protection during hospitalization and outpatient care for India's Elderly

\begin{tabular}{|c|c|c|c|c|c|c|c|c|c|c|}
\hline & \multicolumn{2}{|l|}{ Insurance coverage } & \multicolumn{2}{|c|}{$\begin{array}{l}\text { OOPE in out- } \\
\text { patient care }\end{array}$} & \multicolumn{2}{|c|}{$\begin{array}{l}\text { OOPE during } \\
\text { hospitalization }\end{array}$} & \multicolumn{2}{|c|}{ CHE-10 } & \multicolumn{2}{|c|}{ CHE-25 } \\
\hline & Total insurance coverage & Coverage under PFHI & Pub & Pvt & Pub & Pvt. & Pub & Pvt & Pub & Pvt. \\
\hline Total & 18.9 & 14.3 & 390 & 852 & 6209 & 38,709 & 23.2 & 64.9 & 9.1 & 37.0 \\
\hline \multicolumn{11}{|l|}{ Age group (years) } \\
\hline $60-69$ & 19.3 & 14.5 & 410 & 822 & 5315 & 39,051 & 22.2 & 67.5 & 7.6 & 38.8 \\
\hline 70-79 & 17.8 & 13.9 & 336 & 841 & 8364 & 38,523 & 25.4 & 63.0 & 13.0 & 36.6 \\
\hline 80 and above & 18.7 & 14.3 & 430 & 1039 & 4745 & 37,828 & 23.0 & 58.9 & 6.4 & 30.7 \\
\hline \multicolumn{11}{|l|}{ Place of Residence } \\
\hline Rural & 18.1 & 16.6 & 388 & 816 & 6180 & 32,009 & 25.2 & 67.8 & 10.0 & 40.7 \\
\hline Urban & 20.4 & 9.5 & 394 & 892 & 6268 & 47,200 & 19.0 & 61.3 & 7.1 & 32.4 \\
\hline \multicolumn{11}{|l|}{ Gender } \\
\hline Male & 19.0 & 14.1 & 441 & 857 & 7336 & 44,666 & 25.7 & 67.6 & 10.8 & 41.2 \\
\hline Female & 18.7 & 14.5 & 342 & 847 & 4780 & 31,459 & 20.2 & 61.7 & 6.9 & 31.9 \\
\hline \multicolumn{11}{|l|}{ Marital Status } \\
\hline Never Married & 17.3 & 14.6 & 318 & 1849 & 7857 & 88,010 & 16.6 & 62.0 & 5.7 & 46.6 \\
\hline Currently married & 18.3 & 13.5 & 484 & 861 & 6381 & 40,639 & 21.7 & 67.1 & 9.5 & 38.6 \\
\hline Widowed & 19.9 & 15.8 & 256 & 818 & 4370 & 26,638 & 19.8 & 58.1 & 6.1 & 29.7 \\
\hline \multicolumn{11}{|l|}{ Surviving Children } \\
\hline At least one surviving child & 18.7 & 14.1 & 393 & 849 & 5676 & 36,426 & 20.3 & 63.5 & 8.1 & 35.3 \\
\hline No child & 21.3 & 18.9 & 306 & 937 & 9320 & 56,542 & 40.6 & 77.0 & 14.8 & 51.6 \\
\hline \multicolumn{11}{|l|}{ Social Groups } \\
\hline ST & 22.5 & 20.7 & 246 & 613 & 4102 & 22,546 & 25.2 & 69.2 & 3.5 & 43.3 \\
\hline SC & 15.1 & 13.6 & 451 & 1063 & 7229 & 24,972 & 27.8 & 61.2 & 12.8 & 31.8 \\
\hline $\mathrm{OBC}$ & 20.0 & 16.9 & 324 & 782 & 5523 & 33,098 & 23.6 & 66.8 & 8.9 & 38.8 \\
\hline General & 18.8 & 10.3 & 492 & 860 & 6779 & 48,538 & 19.9 & 64.1 & 8.5 & 36.5 \\
\hline \multicolumn{11}{|l|}{ Education } \\
\hline Illiterate & 17.9 & 16.6 & 333 & 732 & 4409 & 25,878 & 19.7 & 63.5 & 6.4 & 34.3 \\
\hline Up to primary & 19.3 & 14.7 & 363 & 856 & 6065 & 31,672 & 21.9 & 65.2 & 11.6 & 33.9 \\
\hline Up to secondary & 19.4 & 11.8 & 530 & 786 & 6678 & 46,869 & 23.7 & 63.7 & 8.7 & 39.6 \\
\hline Above Secondary & 22.2 & 4.8 & 615 & 1222 & 13,856 & 57,350 & 21.2 & 63.3 & 7.5 & 36.6 \\
\hline \multicolumn{11}{|l|}{ Household occupation } \\
\hline Self employed & 14.9 & 13.0 & 500 & 846 & 6961 & 37,673 & 19.7 & 63.5 & 9.4 & 35.9 \\
\hline Regular Wages & 24.3 & 10.4 & 308 & 1046 & 5998 & 36,478 & 21.9 & 65.2 & 5.3 & 26.3 \\
\hline Casual Labourer & 18.8 & 18.2 & 268 & 618 & 3509 & 22,085 & 23.7 & 63.7 & 8.2 & 36.0 \\
\hline Others & 25.2 & 17.1 & 387 & 830 & 5260 & 42,098 & 21.2 & 63.3 & 8.4 & 43.3 \\
\hline \multicolumn{11}{|l|}{ Economic quintile-Rural } \\
\hline Poorest & 13.5 & 12.8 & 381 & 761 & 5084 & 19,410 & 43.3 & 75.2 & 17.5 & 47.4 \\
\hline Poor & 10.6 & 10.1 & 477 & 908 & 4858 & 28,978 & 24.3 & 67.7 & 4.4 & 45.1 \\
\hline Middle & 19.0 & 18.2 & 360 & 725 & 6293 & 24,807 & 19.3 & 71.4 & 8.7 & 37.8 \\
\hline Rich & 20.9 & 19.6 & 421 & 659 & 5470 & 32,435 & 22.6 & 72.9 & 10.7 & 44.6 \\
\hline Richest & 25.6 & 21.7 & 353 & 918 & 7949 & 39,683 & 22.7 & 60.6 & 9.9 & 36.5 \\
\hline \multicolumn{11}{|l|}{ Economic quintile-Urban } \\
\hline Poorest & 13.4 & 10.4 & 371 & 995 & 5577 & 32,077 & 27.6 & 77.1 & 11.8 & 44.4 \\
\hline Poor & 18.1 & 13.2 & 322 & 862 & 4227 & 41,811 & 21.6 & 63.2 & 6.4 & 34.7 \\
\hline
\end{tabular}


Table 5 Financial Protection during hospitalization and outpatient care for India's Elderly (Continued)

\begin{tabular}{|c|c|c|c|c|c|c|c|c|c|c|}
\hline & \multicolumn{2}{|l|}{ Insurance coverage } & \multicolumn{2}{|c|}{$\begin{array}{l}\text { OOPE in out- } \\
\text { patient care }\end{array}$} & \multicolumn{2}{|c|}{$\begin{array}{l}\text { OOPE during } \\
\text { hospitalization }\end{array}$} & \multicolumn{2}{|c|}{ CHE-10 } & \multicolumn{2}{|c|}{ CHE-25 } \\
\hline & Total insurance coverage & Coverage under PFHI & Pub & Pvt & Pub & Pvt. & Pub & Pvt & Pub & Pvt. \\
\hline Middle & 18.3 & 9.1 & 352 & 845 & 5850 & 42,262 & 13.6 & 63.8 & 3.7 & 34.2 \\
\hline Rich & 19.2 & 9.0 & 441 & 868 & 7091 & 50,633 & 11.6 & 62.3 & 4.9 & 28.2 \\
\hline Richest & 35.6 & 5.5 & 564 & 916 & 13,025 & 60,067 & 11.2 & 48.6 & 6.2 & 26.0 \\
\hline
\end{tabular}

Source: Authors' computation from unit records of NSSO 75th Round 2017-18

years (PAP: 5.9\%, hospitalization rate: 2.4\%) [7]. Selfreported hospitalization rate and PAP were significantly higher in upper socioeconomic population compared to the lower socioeconomic population since perceived healthcare needs are higher in upper socioeconomic population. Various studies have shown that the poor and marginalized sections of society have a higher burden of non-communicable diseases and chronic conditions [17]. In current COVID-19 pandemic, studies from China, Italy, Spain, and the United States have shown that NCD patienst are at higher risk of mortality due to COVID-19 [18-20]. In the elderly population, these comorbid conditions further aggravate the COVID-19 condition and increase mortality [21].

In India, one-third of the elderly went to public healthcare facilities (hospitalization: 39.8\%, outpatient care: $33.6 \%$-Table 2); the remaining two-third went to private healthcare facilities. Often, elderly patients require lifelong treatment, curative and rehabilitative, for their chronic conditions. These conditions require regular follow-up, which includes doctor's consultation, continued medication, and diagnostic tests. India's healthcare systems, both public and private, do not provide the required level of continuity of care, which leads to poor quality of care for the elderly [22]. Rehabilitative care is almost absent in India's public health care system and in some urban areas where private sector provides this care is prohibitively costly, which poor and middle income elderly cannot afford [23]. All these may lead to further suffering, poorer quality of life, and mortality for the elderly.

In the current COVID-19 pandemic, the public healthcare system has been overstretched in handling the avalanche of COVID-19 patients. It has seriously disrupted provision of regular services at public healthcare facilities which include immunization, child and maternal health, dialysis services, emergency surgeries, and general outpatient care [24]. A recent study done by Stop TB Partnership shows that for every month of lockdown in India, there would be an additional 2,32,665 tuberculosis (TB) cases and 71,290 deaths in the period of 2020-2025 [25]. Public facilities are major service providers for the poor and marginalized elderly in society. For instance, $64 \%$ of
ST, $54 \%$ of the casual labourer, and $51 \%$ of rural poorest elderly took inpatient care under the public facility in 2017-18 (refer Table 2).

Private sector, which provides two-thirds of care for the elderly, has not been able to respond adequately in the country [26, 27]. A significant proportion of private providers has either stopped providing care due to fear of the spread of the diseases or have started charging exorbitantly high which cannot be afforded by the poor and middle-class person of the society $[28,29]$.

Availability of regular drugs, related to chronic conditions, face additional logistic constraints after the nation-wide lockdown [30]. As part of the latest controversy on the use of hydroxychloroquine drug for covid19 patients, this drug became unavailable for rheumatic patients, which is a common ailment in the elderly population in India and across the world [31, 32]. Public health facilities, where these drugs are provided free of cost to patients (who are largely poor), reported shortage of regular NCD drugs [33].

Elderly face greater risk of financial hardships due to chronic nature of ailment and comorbidities, which require long term care. Another dimension of financial hardship for the elderly comes from the fact that $70 \%$ are partially or wholly financially dependent on others (refer Table 6). It is even higher for those in the lower socioeconomic groups. For instance, 50\% of elderly males and $90 \%$ of females are dependent on other family members for financial support. In rural areas, elderly parents are dependent on their children who work as a migrant labourer in urban settings. In the current crisis with lockdown, the unemployed, migrants from urban areas to rural areas, would find it difficult to support their families and the elderly dependent parents [34].

One of the direct impacts of the current pandemic for the elderly could be lack of access to food leading to starvation. For instance, a study done by Pradhan shows that $50 \%$ of India's rural households are consuming less food compared to pre-COVID-19 outbreak [35]. Studies across the world also point out that food insecurity, hunger, and malnutrition could beome worse during the pandemic [36].

In terms of social welfare schemes, central and state governments have announced various measures for 
Table 6 Living arrangement and economic independence in elderly population of India

\begin{tabular}{|c|c|c|c|c|c|c|c|c|c|}
\hline \multicolumn{4}{|c|}{ Living arrangements } & \multicolumn{3}{|c|}{ Economic independence } & \multirow{2}{*}{$\begin{array}{l}\text { Physically } \\
\text { immobile }\end{array}$} & \multirow{2}{*}{$\begin{array}{l}\text { Poor } \\
\text { perception } \\
\text { of current } \\
\text { health }\end{array}$} & \multirow{2}{*}{$\begin{array}{l}\text { Perception } \\
\text { of change } \\
\text { in state of } \\
\text { health } \\
\text { being } \\
\text { worse }\end{array}$} \\
\hline $\begin{array}{l}\text { Living with } \\
\text { spouse and } \\
\text { other } \\
\text { members }\end{array}$ & $\begin{array}{l}\text { Living } \\
\text { with } \\
\text { spouse } \\
\text { only }\end{array}$ & $\begin{array}{l}\text { Living without- } \\
\text { spouse but } \\
\text { with children/ } \\
\text { relatives }\end{array}$ & $\begin{array}{l}\text { Living } \\
\text { alone }\end{array}$ & Independent & $\begin{array}{l}\text { Partially } \\
\text { dependent }\end{array}$ & $\begin{array}{l}\text { Fully } \\
\text { dependent }\end{array}$ & & & \\
\hline
\end{tabular}

Total 50.3

Age group (years)

$\begin{array}{lllllllllll}60-69 & 54.9 & 16.0 & 25.1 & 4.1 & 35.0 & 23.9 & 41.2 & 4.5 & 13.6 & 16.2 \\ 70-79 & 44.2 & 11.5 & 39.9 & 4.5 & 23.1 & 22.5 & 54.4 & 9.3 & 26.8 & 26.3 \\ \begin{array}{l}80 \text { and } \\ \text { above }\end{array} & 31.7 & 7.3 & 57.1 & 4.0 & 12.3 & 17.0 & 70.8 & 27.5 & 46.0 & 43.7\end{array}$

Place of Residence

$\begin{array}{lllllllllll}\text { Rural } & 51.1 & 13.4 & 31.1 & 4.4 & 28.5 & 24.5 & 47.0 & 7.6 & 21.4 & 22.8 \\ \text { Urban } & 48.6 & 15.5 & 32.2 & 3.7 & 33.3 & 19.7 & 47.0 & 7.5 & 16.1 & 17.3\end{array}$

Gender

$\begin{array}{llll}\text { Male } & 64.1 & 17.9 & 16.4 \\ \text { Female } & 37.0 & 10.4 & 46.0\end{array}$

Female
Marital Status

Never $\quad 4.6$
Married/
Currently 74.8
married
Widowed

Surviving Children

$$
\begin{aligned}
& \text { one child } \\
& \text { No child } 8.9 \\
& \text { Social Groups }
\end{aligned}
$$$$
\begin{array}{ll}
\text { ST } & 48.7 \\
\text { SC } & 51.1
\end{array}
$$

SC $\quad 51.1$

$\begin{array}{ll}3.6 & 69.6 \\ 21.7 & 3.3 \\ 0.2 & 83.4\end{array}$

$\begin{array}{ll}1.6 & 50.9 \\ 6.6 & 100\end{array}$

83.4

$\begin{array}{ll}\mathrm{OBC} & 48.3 \\ \text { General } & 52.6\end{array}$

$12.8 \quad 31.5$
43.5

$\begin{array}{ll}22.2 & 37.3 \\ 0.3 & 38.3 \\ 11.0 & 14.5\end{array}$

Education

$\begin{array}{ll}\text { Illiterate } & 44.9 \\ \text { Up to } & 54.0 \\ \text { primary } & \\ \text { Up to } & 60.1 \\ \text { secondary } & \\ \text { Above } & 57.0 \\ \text { Secondary } & \end{array}$

$\begin{array}{ll}12.6 & 34.5 \\ 14.4 & 30.2 \\ 13.1 & 34.3 \\ 15.4 & 28.0\end{array}$

$\begin{array}{ll}3.6 & 29.1 \\ 16.1 & 53.1\end{array}$

$\begin{array}{ll}11.3 & 38.8 \\ 13.2 & 29.4\end{array}$

$\begin{array}{ll}4.2 & 25.7 \\ 4.2 & 29.0 \\ 4.3 & 28.5 \\ 4.0 & 33.4\end{array}$

$\begin{array}{ll}17.8 & 19.8 \\ 25.4 & 13.8\end{array}$

$\begin{array}{ll}5.0 & 21.0 \\ 3.4 & 29.5\end{array}$

22.9
23.0

\begin{tabular}{|c|c|c|c|c|c|c|c|c|c|c|}
\hline $\begin{array}{l}\text { Self } \\
\text { employed }\end{array}$ & 58.0 & 9.2 & 32.1 & 0.8 & 30.3 & 22.6 & 47.1 & 7.3 & 18.9 & 21.1 \\
\hline $\begin{array}{l}\text { Regular } \\
\text { Wages }\end{array}$ & 56.3 & 3.5 & 39.7 & 0.5 & 21.8 & 26.0 & 52.2 & 9.3 & 17.5 & 18.4 \\
\hline $\begin{array}{l}\text { Casual } \\
\text { Labourer }\end{array}$ & 48.3 & 10.2 & 38.5 & 3.1 & 26.7 & 25.9 & 47.4 & 7.9 & 22.0 & 22.8 \\
\hline
\end{tabular}

$\begin{array}{llll}26.1 & 6.2 & 17.4 & 19.6 \\ 67.1 & 8.9 & 21.7 & 22.4\end{array}$

Secondary
Household occupation

Economic quintile-Rural

$$
\text { Poorest } 55.3
$$


Table 6 Living arrangement and economic independence in elderly population of India (Continued)

\begin{tabular}{|c|c|c|c|c|c|c|c|c|c|c|}
\hline & \multicolumn{4}{|c|}{ Living arrangements } & \multicolumn{3}{|c|}{ Economic independence } & \multirow{2}{*}{$\begin{array}{l}\text { Physically } \\
\text { immobile }\end{array}$} & \multirow{2}{*}{$\begin{array}{l}\text { Poor } \\
\text { perception } \\
\text { of current } \\
\text { health }\end{array}$} & \multirow{2}{*}{$\begin{array}{l}\text { Perception } \\
\text { of change } \\
\text { in state of } \\
\text { health } \\
\text { being } \\
\text { worse }\end{array}$} \\
\hline & $\begin{array}{l}\text { Living with } \\
\text { spouse and } \\
\text { other } \\
\text { members }\end{array}$ & $\begin{array}{l}\text { Living } \\
\text { with } \\
\text { spouse } \\
\text { only }\end{array}$ & $\begin{array}{l}\text { Living without- } \\
\text { spouse but } \\
\text { with children/ } \\
\text { relatives }\end{array}$ & $\begin{array}{l}\text { Living } \\
\text { alone }\end{array}$ & Independent & $\begin{array}{l}\text { Partially } \\
\text { dependent }\end{array}$ & $\begin{array}{l}\text { Fully } \\
\text { dependent }\end{array}$ & & & \\
\hline Poor & 54.2 & 6.0 & 36.7 & 3.1 & 25.4 & 25.3 & 49.3 & 7.6 & 22.3 & 20.9 \\
\hline Middle & 53.7 & 12.3 & 31.5 & 2.5 & 27.0 & 25.7 & 47.4 & 6.4 & 19.5 & 24.5 \\
\hline Rich & 49.2 & 14.5 & 31.4 & 4.8 & 29.1 & 23.5 & 47.4 & 7.2 & 21.3 & 21.8 \\
\hline Richest & 43.6 & 22.7 & 26.7 & 7.0 & 34.2 & 20.5 & 45.3 & 8.3 & 22.1 & 24.1 \\
\hline \multicolumn{11}{|c|}{ Economic quintile-Urban } \\
\hline Poorest & 54.2 & 6.6 & 35.9 & 3.2 & 26.3 & 25.4 & 48.4 & 8.5 & 16.5 & 20.4 \\
\hline Poor & 56.0 & 7.5 & 34.3 & 2.3 & 30.6 & 19.5 & 49.9 & 7.3 & 16.9 & 17.9 \\
\hline Middle & 50.4 & 12.6 & 34.7 & 2.3 & 30.6 & 18.5 & 50.8 & 5.9 & 17.4 & 17.3 \\
\hline Rich & 45.8 & 22.2 & 27.8 & 4.3 & 35.9 & 18.6 & 45.5 & 6.8 & 14.1 & 15.1 \\
\hline Richest & 35.6 & 29.8 & 28.2 & 6.5 & 45.1 & 15.7 & 39.3 & 9.5 & 15.5 & 15.6 \\
\hline
\end{tabular}

Source: Authors' computation from unit records of NSSO 75th Round 2017-18

delivering free food grains and direct transfer of pension for the elderly people [37]. However, there is considerable variations in old age pension across states of India. At all India level, only $29.6 \%$ of the elderly receive an old-age pension of the total older population [38]. Evidently, current COVID-19 situation makes the elderly more vulnerable financially.

Physical/social distancing has considerably increased social isolation and more so for the elderly population [39]. Studies have established that social isolation increases depression, suicidality, and a higher chance of increased inflammatory response in the elderly [40, 41]. This will also exacerbate the vulnerability of the elderly who are already suffering from psychiatric or neurological conditions $(8.2 \%$ of total hospitalization in the last 365 days and $4.4 \%$ of PAP in last 15 days). For instance, recent studies published post-COVID-19 outbreak has shown that this pandemic has considerably increased the vulnerability of demented patients and their caregivers across the world [42, 43]. One of the suggestions which have been given to elderly by Ministry of Health and Family Welfare (MoHFW), Government of India [44], and Centre for Diseases Control and Prevention (CDC), Atlanta, United States [45], is to be in contact with significant others in current COVID-19 outbreak, through mobile phone and video call. However, a significant proportion of the elderly population finds it difficult to operate a mobile phone which has become one of the essential skills required for daily living [46]. In India, 4.2\% (50 lakhs) of elderly live alone, and it was more so for women (6.6\%), never married elderly $(22.2 \%)$, elderly without children $(16.1 \%)$ and illiterate (5.0\%).
Among the elderly, $7.6 \%$ are immobile, and it is even more so among the poorest (rural: $8.4 \%$, urban: $8.5 \%$ ), illiterate (8.7\%), and widowed (11.3\%). Often, they are taken care of by a family member; care provision through a hired provider is rare. In current social distancing measures, it has adversely impacted the caregiving for these elderly. Studies also show that family members (as caregivers) have been associated with an elevated level of depression and anxiety, higher use of psychoactive medication, poorer self-reported physical health, compromised immune function, and increased risk of early death [47].

This unprecedented situation of COVID-19 draws our attention towards the need for strengthening public healthcare facilities in the country. The public healthcare system is largely managing the pandemic across the country, despite several weaknesses. COVID-19 is a wake-up call for greater investment in public health facilities which include strengthening public infrastructure, skill building for health professionals, strengthening diseases surveillance system, improving quality of care in public healthcare facilities, and better continuity of care between primary and tertiary care. The idea of "Health and Wellness Centre (HWCs)" under Ayushman Bharat is a welcome decision since it proposes to provide comprehensive primary healthcare at health sub-centre (HSC), or nearer home. The elderly population and poor will be the major beneficiaries of this scheme since they bear a higher burden of diseases and ill-health. Current COVID-19 pandemic shows the need of implementing the HWCs scheme [48].

One of the limitations of this study is that it uses 2017-18 data and not the real-time data collected 
Table 7 Variation in various indicators of elderly health from 71st Round NSS, 2014, to 75th Round NSS, 2017-18

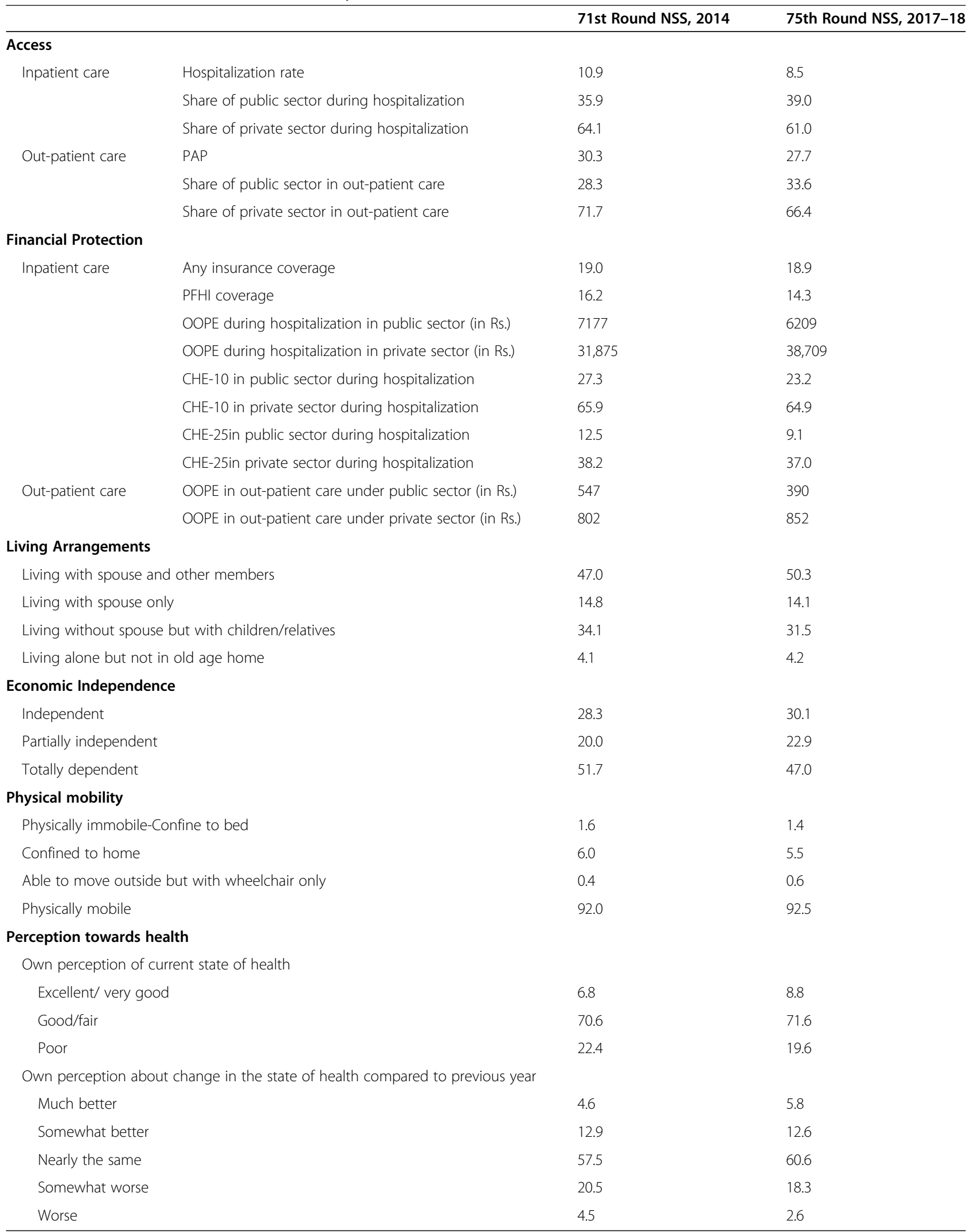


during the COVID-19 outbreak. Though the national sample surveys throw considerable light on the vulnerability of the elderly, we need a deeper understanding of their lived experiences and coping mechanisms during the covid-19 outbreak. Such studies would provide deeper sociological insights required for more "responsive" policy measures to enhance the quality of life of the elderly.

\section{Conclusions}

The current COVID-19 pandemic poses a greater risk of social isolation among the elderly, which may lead to greater adverse health impact. The poor among the elderly has suffered more than others. As a result, their access to regular primary healthcare services, and continuity of care that is essential for those suffering from non-communicable diseases, given their dependency and lack of mobility, may have worsened further during this pandemic. Overall, given the evidence on the possible hardships that the elderly may be have already gone through during the pandemic and hardships that they may face in the future, the importance of strengthening public health care system cannot be over-emphasized. We urge a much greater investment by government to mitigate adverse impact of the pandemic and enhance the quality of life of the elderly in the future.

\section{Supplementary information}

Supplementary information accompanies this paper at https://doi.org/10. 1186/s12992-020-00619-7.

Additional file 1: Table S1. Factors affecting hospitalization in India's elderly, 2017-18. Table S2. Factors affecting PAP in India's elderly, 201718. Table S3. Factors affecting catastrophic health expenditure at 10\% threshold (CHE-10) in India's elderly, 2017-18. Table S4. Factors affecting catastrophic health expenditure at 25\% threshold (CHE-25) in India's elderly, 2017-18. Table S5. Factors affecting 'living alone' in India's elderly, 2017-18. Table S6. Factors affecting economic dependence in India's elderly, 2017-18.

\section{Abbreviations}

CHE: Catastrophic health expenditure: CHE-10: Proportion of households in a population who face catastrophic health expenditure computed using the threshold of $10 \%$ of usual annual consumption expenditure; $\mathrm{CHE}$ -

25: Proportion of households in a population who face catastrophic health expenditure computed using the threshold of $25 \%$ of usual annual consumption expenditure.; HSC: Health Sub Centre; HWC: Health and Wellness Centre; NSS: National sample survey; NSSO: National sample survey office; OOPE: Out-of-pocket expenditure; PFHI: Public Funded Health Insurance; PHC: Primary Health Centre; UAPCE: Usual annual per capita expenditure; UMPCE: Usual monthly per capita expenditure

\section{Acknowledgements}

Not applicable.

\section{Authors' contributions}

RA and VRM contributed to the study design, to the interpretation of results, and writing the manuscript. RA analyzed the data and VRM verified the results. Both authors read and approved the final version of the manuscript.
Funding

None.

Availability of data and materials

The present study is based on India's National Sample Survey, 2017-18, which is freely available in the public domain (http://www.mospi.gov.in/unitlevel-data-report-nss-75th-round-july-2017-june-2018-schedule-250socialconsumption-health) [7].

Ethics approval and consent to participate

The data analysed for this article are from the National Sample Survey, 75th Round, which contains anonymised data in the public domain (online available). The researchers had no access to personal identifiable data. The survey is undertaken by the National Sample Survey Office (NSSO) of the Ministry of Statistics and Programme Implementation of the Government of India. Data available in public domain are approved for use for research purpose by Ministry of Statistics and Programme Implementation, Government of India [7]

Consent for publication

Not Applicable.

\section{Competing interests}

The author declares there was competing interest.

Received: 9 July 2020 Accepted: 16 September 2020

Published online: 08 October 2020

\section{References}

1. Armitage R, Nellums LB. COVID-19 and the consequences of isolating the elderly. Lancet Public Health. 2020;0. https://doi.org/10.1016/S24682667(20)30061-X.

2. Onder G, Rezza G, Brusaferro S. Case-fatality rate and characteristics of patients dying in relation to COVID-19 in Italy. JAMA. 2020. https://doi.org/ 10.1001/jama.2020.4683.

3. Guan W, Liang W, Zhao Y, Liang H, Chen Z, Li Y, et al. Comorbidity and its impact on 1590 patients with Covid-19 in China: a Nationwide analysis. Eur Respir J. 2020. https://doi.org/10.1183/13993003.00547-2020.

4. World In Data. Mortality Risk of COVID-19 - Statistics and Research. Our World in Data. 2020. https://ourworldindata.org/mortality-risk-covid. Accessed 10 Jun 2020.

5. Yancy CW. COVID-19 and African Americans. JAMA. 2020;323:1891-2.

6. Shelar J, Mahale A. Coronavirus in Dharavi I when a virus finds space in India's largest slum. The Hindu 2020. https://www.thehindu.com/news/ cities/mumbai/when-a-virus-finds-space-in-indias-largest-slum/article3153 7623.ece.

7. Government of India. NSS 75th Round-Key Indicators of Social Consumption in India: Health. New Delhi; 2019. http://www.mospi.gov.in/unit-level-datareport-nss-75th-round-july-2017-june-2018-schedule-250social-consumptionhealth. Accessed 12 Feb 2020.

8. Das J, Hammer J, Sánchez-Paramo C. The impact of recall periods on reported morbidity and health seeking behavior. J Dev Econ. 2012;98:76-88.

9. WHO. Tracking universal health coverage: 2017 Global monitoring report: WHO; 2017. http://www.who.int/healthinfo/universal_health_coverage/ report/2017/en/. Accessed 10 Mar 2019

10. Government of India. Report on NSS 76th Round, Persons with Disabilities in India, 2018. New Delhi; 2019. http://www.mospi.gov.in/sites/default/files/ NSS7626d/Report 583 Final.pdf.

11. Ranjan A, Dixit P, Mukhopadhyay I, Thiagarajan S. Effectiveness of government strategies for financial protection against costs of hospitalization Care in India. BMC Public Health. 2018;18:501.

12. Agrawal S. Effect of living arrangement on the health status of elderly in India: findings from a national cross sectional survey. Asian Popul Stud. 2012;8:87-101.

13. Bloom BE, Covington S. Addressing the mental health needs of women offenders. Women's mental health issues across the criminal justice system; 2008. p. 160-76.

14. Kumar S, Kumar KA. Living arrangement and economic dependency among the elderly in India: a comparative analysis of EAG and non EAG states. Ageing Int. 2019;44:352-70. 
15. Dean A, Kolody B, Wood P, Matt GE. The influence of living alone on depression in elderly persons. J Aging Health. 1992;4:3-18.

16. Wang H, Chen K, Pan Y, Jing F, Liu H. Associations and impact factors between living arrangements and functional disability among older Chinese adults. PLoS One. 2013;8:e53879.

17. Bhojani U, Beerenahalli TS, Devadasan R, Munegowda CM, Devadasan N, Criel B, et al. No longer diseases of the wealthy: prevalence and healthseeking for self-reported chronic conditions among urban poor in southern India. BMC Health Serv Res. 2013;13:306.

18. The Novel Coronavirus Pneumonia Emergency Response Epidemiology Team. The epidemiological characteristics of an outbreak of 2019 novel coronavirus diseases (COVID-19) - China, 2020. 2020. http://weekly. chinacdc.cn/en/article/id/e53946e2-c6c4-41e9-9a9b-fea8db1a8f51. Accessed 15 Mar 2020.

19. Kluge HHP, Wickramasinghe $\mathrm{K}$, Rippin $\mathrm{HL}$, Mendes $\mathrm{R}$, Peters $\mathrm{DH}$, Kontsevaya $A$, et al. Prevention and control of non-communicable diseases in the COVID-19 response. Lancet. 2020;0. https://doi.org/10.1016/S01406736(20)31067-9.

20. Goumenou M, Sarigiannis D, Tsatsakis A, Anesti O, Docea AO, Petrakis D, et al. COVID-19 in northern Italy: an integrative overview of factors possibly influencing the sharp increase of the outbreak. Mol Med Rep. 2020;22:20-32.

21. Jordan RE, Adab P, Cheng KK. Covid-19: risk factors for severe disease and death. Bri Med J; 2020.368:m1198.

22. Kumar SG, Roy G, Kar SS. Disability and rehabilitation Services in India: issues and challenges. J Family Med Prim Care. 2012;1:69-73.

23. Ranjan A, Sundararaman T, Garg S, Debashish D. Progress towards universal health coverage in context of rheumatic diseases in India. Int J Rheum Dis. 2019;22(5):880-9.

24. Cash R, Patel V. Has COVID-19 subverted global health? Lancet 2020;0. doi: https://doi.org/10.1016/S0140-6736(20)31089-8.

25. Stop TB Partnership. The potential impact of the covid-19 response on tuberculosis in high-burden countries: A modelling analysis. 2020. http:// www.stoptb.org/assets/documents/news/Modeling\%20Report_1\%20May\%2 02020_FINAL.pdf.

26. Krishnan A, Dasgupta R. Science, policy, people, and public health: what is COVID-19 teaching us? Indian J Public Health. 2020;64:87.

27. Sundararaman T. Health systems preparedness for COVID-19 pandemic. Indian J Public Health. 2020;64:91.

28. Bedi A. Delhi private hospitals refusing medical care due to Covid-19 will lose registration: AAP govt. 2020. https://theprint.in/health/delhi-privatehospitals-refusing-medical-care-due-to-covid-19-will-lose-registration-aapgovt/402914/. Accessed 6 May 2020.

29. Yamunan S. Fear of Covid-19 spread makes private hospitals turn away patients - or charge them higher bills. Scroll.in. 2020. https://scroll.in/article/ 959727/fear-of-covid-19-spread-makes-private-hospitals-turn-away-patientsor-charge-them-higher-bills. Accessed 12 May 2020.

30. Mohanty D, Kumar R, Apparasu S. National lockdown over Covid-19 leads to drug shortage for HIV patients. Hindustan Times. 2020. https://www. hindustantimes.com/india-news/national-lockdown-leads-to-drug-shortagefor-hiv-patients/story-81uSkJbhDACZzbkAmZSsjO.html. Accessed 6 May 2020.

31. Khaira R. Sudden Hydroxycholoroquine shortage is forcing Indian doctors to make tough choices. 2020. https://www.huffingtonpost.in/entry/ hydroxychloroquine-shortage-covid-coronavirus_in_5e8f4b6ac5b6b371812 d5b02. Accessed 6 May 2020.

32. Mehta B, Salmon J, Ibrahim S. Potential shortages of Hydroxychloroquine for patients with lupus during the coronavirus disease 2019 pandemic. JAMA Health Forum. 2020;1:e200438.

33. Kumar A, Rajasekharan Nayar K, Koya SF. COVID-19: Challenges and its consequences for rural health care in India. Public Health Pract. 2020; 100009:1-2

34. The Economist. Covid stops many migrants sending money home. De Economist. 2020; https://www.economist.com/middle-east-and-africa/2020/ 04/16/covid-stops-many-migrants-sending-money-home. Accessed $27 \mathrm{Apr}$ 2020.

35. Hindustant Times. Covid-19 lockdown: 50 percent of surveyed households in rural India eating less. Hindustan Times 2020. https://www. hindustantimes.com/india-news/covid-19-lockdown-50-percent-of-surveyedhouseholds-in-rural-india-eating-less/story-DZZOQFIWPq7k1AZ2Gu7f0M. html. Accessed 13 May 2020
36. The Lancet Global Health. Food insecurity will be the sting in the tail of COVID-19. Lancet Glob Health. 2020;8:e737.

37. Ministry of Finance. More than Rs 36,659 crore transferred by using Direct Benefit Transfer (DBT) through Public Financial Management System (PFMS)in the Bank accounts of 16.01 crore beneficiaries during COVID 2019 lockdown. 2020. pib.gov.in/Pressreleaseshare.aspx?PRID=1616022. Accessed 12 Jun 2020.

38. Goli S, Reddy AB, James KS, Srinivasan V. Economic Independence and social security among India's elderly. Econ Polit Wkly. 2019;54:32-41.

39. Jawaid A. Protecting older adults during social distancing. Science. 2020;368:145.

40. Santini ZI, Jose PE, Cornwell EY, Koyanagi A, Nielsen L, Hinrichsen C, et al. Social disconnectedness, perceived isolation, and symptoms of depression and anxiety among older Americans (NSHAP): a longitudinal mediation analysis. Lancet Public Health. 2020;5:e62-70.

41. Cole SW, Capitanio JP, Chun K, Arevalo JM, Ma J, Cacioppo JT. Myeloid differentiation architecture of leukocyte transcriptome dynamics in perceived social isolation. Proc Natl Acad Sci. 2015;112:15142-7.

42. Wang H, Li T, Barbarino P, Gauthier S, Brodaty H, Molinuevo JL, et al. Dementia care during COVID-19. Lancet. 2020;395:1190-1.

43. The Economist. For people with dementia, the coronavirus pandemic is a nightmare. De Economist. 2020; https://www.economist.com/international/2 020/04/26/for-people-with-dementia-the-coronavirus-pandemic-is-anightmare. Accessed 27 Apr 2020.

44. Government of India. Health Advisory for Elderly Population of India during COVID19. 2020. https:/www.mohfw.gov.in/pdf/AdvisonyforElderlyPopulation.pdf.

45. CDC. Coronavirus Disease 2019 (COVID-19). Centers for Disease Control and Prevention. 2020. https://www.cdc.gov/coronavirus/2019-ncov/daily-lifecoping/managing-stress-anxiety.html. Accessed 4 May 2020.

46. Navabi N, Ghaffari F, Jannat-Alipoor Z. Older adults' attitudes and barriers toward the use of mobile phones. Clin Interv Aging. 2016;11:1371-8.

47. CDC. Caregiving. 2019. https://www.cdc.gov/aging/caregiving/index.htm. Accessed 6 May 2020

48. Govt. of India. National Health Policy. 2017:2017 https://mohfw.gov.in/ documents/policy. Accessed 18 Mar 2019.

\section{Publisher's Note}

Springer Nature remains neutral with regard to jurisdictional claims in published maps and institutional affiliations.

Ready to submit your research? Choose BMC and benefit from:

- fast, convenient online submission

- thorough peer review by experienced researchers in your field

- rapid publication on acceptance

- support for research data, including large and complex data types

- gold Open Access which fosters wider collaboration and increased citations

- maximum visibility for your research: over $100 \mathrm{M}$ website views per year

At BMC, research is always in progress.

Learn more biomedcentral.com/submissions 